\title{
Rotational motion of a droplet induced by interfacial tension
}

\author{
Ken H. Nagai* \\ Department of Physics, Graduate School of Science, \\ The University of Tokyo, Tokyo, 113-0033, Japan \\ Fumi Takabatake and Masatoshi Ichikawa \\ Department of Physics, Kyoto University, Kyoto, 606-8502, Japan \\ Yutaka Sumino \\ Department of Physics, Faculty of Education, \\ Aichi University of Education, Aichi 448-8542, Japan \\ Hiroyuki Kitahata \\ Department of Physics, Graduate School of Science, \\ Chiba University, Chiba 263-8522, Japan and \\ PRESTO, Japan Science and Technology Agency, Saitama, 332-0012, Japan \\ Natsuhiko Yoshinaga \\ WPI-AIMR, Tohoku University, Sendai 980-8577, Japan
}

(Dated: July 7, 2018)

\begin{abstract}
Spontaneous rotation of a droplet induced by the Marangoni flow is analyzed in a two-dimensional system. The droplet with the small particle which supplies a surfactant at the interface is considered. We calculated flow field around the droplet using Stokes equation and found that advective nonlinearity breaks symmetry for rotation. Theoretical calculation indicates that the droplet spontaneously rotates when the radius of the droplet is an appropriate size. The theoretical results were validated through comparison with the experiments.

PACS numbers: 47.55.D-, 47.63.mf, 68.03.Cd
\end{abstract}

\footnotetext{
*nagai@daisy.phys.s.u-tokyo.ac.jp
} 


\section{INTRODUCTION}

Recently, self-propelled motion of both biological objects and nonbiological objects are extensively studied to find general aspects in self-propelled systems [1]. Collective behaviors such as nonequilibrium order-disorder transition of direction of motion are candidates for such general characteristics [2]. One of the best experimental systems to investigate general aspects is self-propelled objects driven by interfacial tension [3, 4]. Actually, several results on collective behavior of the objects driven by interfacial tension have been reported recently [5]. This is because this kind of systems usually consists of a small number of elements and allows us to perform experiments under broad parameter space. Despite of this advantages, however, mechanical analysis for above droplet motion is still lacking.

The above-described droplet motion driven by interfacial tension is observed even under isotropic conditions. Although a droplet itself has no asymmetry, symmetry of interfacial tension is broken through the advective nonlinearity. Actually, Toyota et al. reported spontaneous translational motion of a circular droplet [4]. Recently, theoretical analysis for such translational motion has been reported and the nonlinear effect which breaks the symmetry has been made clear [6]. Not only spontaneous translational motion but also spontaneous rotation has also been demonstrated by Takabatake et al. experimentally [7]. In this system, a droplet rotates even though the system is mirror symmetric. Although this system was analyzed using Langevin equation in the previous study, mechanical analysis is lacking and the physical mechanism of symmetry breaking is not clear. In this article, we analyzed the spontaneous rotation of a droplet using an advection-diffusion equation coupled with Stokes equation. We found that the droplet can rotate when the droplet has an appropriate radius. To validate our model, theoretical results were compared with the experimental ones.

\section{MODEL EQUATION}

We consider a circular undeformable oil droplet with a radius, $R$, in an aqueous phase in a two-dimensional space, as shown in Fig. 1(a). A small solid particle is attached at the interface and a surfactant that reduces interfacial tension is supplied to both the inside and the interface of the droplet from the particle. In the droplet, the surfactant is decomposed. 
(a)

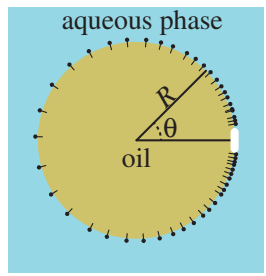

(b-1)

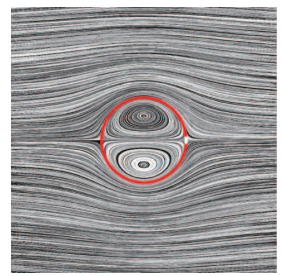

(b-2)

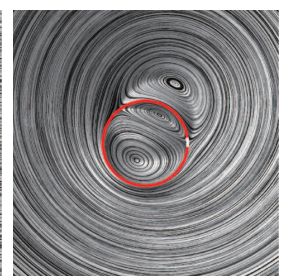

FIG. 1. (Color Online) (a) Schematic diagram of the model in this article. (b) The flow field when the interface concentration field is $c(\theta)$. The images are stream line around the droplet in the frame where the particle and the center of mass of the droplet are fixed. The red line is the oil-water interface and the white line is the particle. (b-1) is the flow field when $c(\theta)=1+0.1 \cos \theta$ (symmetric concentration field) and (b-2) is the flow field when $c(\theta)=1+0.2 \cos \theta+0.1 \sin \theta$ (asymmetric concentration field).

As a result of these process, there appears inhomogeneity of interfacial tention, and the droplet is driven by the Marangoni flow induced by the interfacial tension gradient. In a laboratory frame, the particle can freely move along the interface. Without loss of generality, we may also consider a frame in which the particle and the center of mass of the droplet are fixed. Polar coordinates are introduced with the center of mass of the droplet regarded as the origin. The position of the particle is fixed at $r=R$ and $\theta=0$. With the assumption of low Reynolds number, the velocity field is calculated using Stokes equation as follows,

$$
-\nabla p+\eta \nabla^{2} \boldsymbol{v}=\mathbf{0}
$$

where $\boldsymbol{v}$ is the velocity, $p$ is the pressure field and $\eta$ is the viscosity coefficient. In our model, $\boldsymbol{v}$ satisfies the incompressive condition, $\boldsymbol{\nabla} \cdot \boldsymbol{v}=0$. Due to the interfacial gradient, there is the following stress jump at the interface [8]:

$$
\left.\sigma_{r \theta}^{(\mathrm{i})}\right|_{r=R}=\left.\sigma_{r \theta}^{(\mathrm{o})}\right|_{r=R}+\frac{1}{R} \frac{\partial \gamma}{\partial \theta}
$$

where $\boldsymbol{\sigma}$ is the stress tensor, the superscripts "(i)" and "(o)" correspond to the inside and outside of the droplet, and $\gamma(\theta)$ is the interfacial tension between two fluids. Here, $\gamma$ linearly depends on the interface concentration of the surfactant, $c(\theta)=$ $c^{0}+\sum_{m=1}^{\infty}\left(c_{m}^{\mathrm{c}} \cos m \theta+c_{m}^{\mathrm{s}} \sin m \theta\right)$, as $\gamma(\theta)=\gamma_{0}-k c(\theta)$.

Assuming that the surfactant supplied from the particle is distributed only at the interface 
due to fast decomposition in the bulk, the time evolution of $c(\theta)$ can be described as

$$
\frac{\partial c}{\partial t}+\frac{u}{R} \frac{\partial c}{\partial \theta}=\frac{D}{R^{2}} \frac{\partial^{2} c}{\partial \theta^{2}}-\alpha c+\pi \alpha \beta \delta(\theta)
$$

where $D$ is the diffusion constant at the interface, $\alpha$ is the decomposition rate, and $\pi \alpha \beta$ is the rate of supply. In Eq. (3), the stationary flow field satisfying Eq. (1) is used as $u$, which is determined by $c(\theta)$.

\section{SOLUTION OF THE TWO DIMENSIONAL STOKES EQUATION}

In this section, $\boldsymbol{v}$ satisfying Eq. (1) is calculated under given concentration field at the interface, $c(\theta)$. In the polar coordinate, $\boldsymbol{v}$ is described as

$$
\boldsymbol{v}=v_{r}(r, \theta) \boldsymbol{e}_{r}+v_{\theta}(r, \theta) \boldsymbol{e}_{\theta},
$$

where $\boldsymbol{e}_{r}$ and $\boldsymbol{e}_{\theta}$ are the unit vectors in the radial and angular directions, respectively. Due to incompressibility,

$$
\boldsymbol{\nabla} \cdot \boldsymbol{v}=\frac{1}{r} \frac{\partial}{\partial r}\left(r v_{r}\right)+\frac{1}{r} \frac{\partial v_{\theta}}{\partial \theta}=0
$$

therefore, using the stream function, $\Psi$, the solution of Eq. (1) can be described as

$$
\begin{aligned}
& v_{r}=\frac{1}{r} \frac{\partial \Psi}{\partial \theta} \\
& v_{\theta}=-\frac{\partial \Psi}{\partial r} .
\end{aligned}
$$

Taking rotation of both sides of Eq. (1) and substituting Eqs. (6) and (7), we can obtain

$$
\nabla^{2} \nabla^{2} \Psi=0
$$

where

$$
\nabla^{2}=\frac{1}{r} \frac{\partial}{\partial r}\left(r \frac{\partial}{\partial r}\right)+\frac{1}{r^{2}} \frac{\partial^{2}}{\partial \theta^{2}}
$$


The solutions inside the droplet, $\Psi^{(\mathrm{i})}$, and the outside the droplet, $\Psi^{(\mathrm{o})}$, are

$$
\begin{aligned}
\Psi^{(\mathrm{i})}(r, \theta)= & B_{0}^{(\mathrm{i})}\left(\frac{r}{R}\right)^{2} \\
& +\left(A_{1}^{\mathrm{c}}\left(\frac{r}{R}\right)^{3}+C_{1}^{\mathrm{c}(\mathrm{i})} \frac{r}{R}\right) \cos \theta \\
& +\left(A_{1}^{\mathrm{s}}\left(\frac{r}{R}\right)^{3}+C_{1}^{\mathrm{s}(\mathrm{i}))} \frac{r}{R}\right) \sin \theta \\
& +\sum_{m=2}^{\infty}\left\{\left(A_{m}^{\mathrm{c}}\left(\frac{r}{R}\right)^{m+2}+C_{m}^{\mathrm{c}}\left(\frac{r}{R}\right)^{m}\right) \cos m \theta\right. \\
& \left.+\left(A_{m}^{\mathrm{s}}\left(\frac{r}{R}\right)^{m+2}+C_{m}^{\mathrm{s}}\left(\frac{r}{R}\right)^{m}\right) \sin m \theta\right\} \\
\Psi^{(\mathrm{o})}(r, \theta)= & B_{0}^{(\mathrm{o})}\left(\frac{r}{R}\right)^{2}+C_{0} \ln \frac{r}{R} \\
& +\left(B_{1}^{\mathrm{c}} \frac{r}{R} \ln \frac{r}{R}+C_{1}^{\mathrm{c}(\mathrm{o})} \frac{r}{R}+D_{1}^{\mathrm{c}} \frac{R}{r}\right)^{\cos \theta} \\
& +\left(B_{1}^{\mathrm{s}} \frac{r}{R} \ln \frac{r}{R}+C_{1}^{\mathrm{s}(\mathrm{o})} \frac{r}{R}+D_{1}^{\mathrm{s}} \frac{R}{r}\right)^{\sin \theta} \\
& +\sum_{m=2}^{\infty}\left\{\left(B_{m}^{\mathrm{c}}\left(\frac{R}{r}\right)^{m-2}+D_{m}^{\mathrm{c}}\left(\frac{R}{r}\right)^{m}\right) \cos m \theta\right. \\
& +\left(B_{m}^{\mathrm{s}}\left(\frac{R}{r}\right)^{m-2}+D_{m}^{\mathrm{s}}\left(\frac{R}{r}\right)^{m}\right) \sin m \theta . \\
& \left(\frac{m}{R}\right)
\end{aligned}
$$

The boundary conditions of flow field at the interface are $\left.v_{r}^{(\mathrm{o})}\right|_{r=R}=\left.v_{r}^{(i)}\right|_{r=R}=0$ and $\left.v_{\theta}^{(\mathrm{o})}\right|_{r=R}=\left.v_{\theta}^{(i)}\right|_{r=R}$ since the droplet shape is fixed. Since there is no external force, a torque free condition,

$$
\boldsymbol{T}=\int \mathrm{d} l\left(\left.\boldsymbol{n} \cdot \boldsymbol{\sigma}^{(\mathrm{o})}\right|_{r=R}\right) \times \boldsymbol{r}=\mathbf{0}
$$

and a force free condition,

$$
\boldsymbol{f}=\left.\int \mathrm{d} l \boldsymbol{n} \cdot \boldsymbol{\sigma}^{(\mathrm{o})}\right|_{r=R}=\mathbf{0}
$$

have to be satisfied, where $l$ is the distance along the interface. Using the conditions de- 
scribed above and Eq. (2), the coefficients are calculated as

$$
\begin{aligned}
B_{0}^{(\mathrm{i})}= & B_{0}^{(\mathrm{o})}, \\
C_{0}= & 0, \\
B_{1}^{\mathrm{c}}= & B_{1}^{\mathrm{s}}=0, \\
A_{1}^{\mathrm{c}}= & -C_{1}^{\mathrm{c}(\mathrm{i})}=C_{1}^{\mathrm{c}(\mathrm{o})}=-D_{1}^{\mathrm{c}}=\frac{\kappa c_{m}^{\mathrm{s}} R}{2}, \\
A_{1}^{\mathrm{s}}= & -C_{1}^{\mathrm{s}(\mathrm{i})}=C_{1}^{\mathrm{s}(\mathrm{o})}=-D_{1}^{\mathrm{s}}=-\frac{\kappa c_{m}^{\mathrm{c}} R}{2}, \\
A_{m}^{\mathrm{c}}= & B_{m}^{\mathrm{c}}=-C_{m}^{\mathrm{c}}=-D_{m}^{\mathrm{c}}=\frac{\kappa c_{m}^{\mathrm{s}} R}{2} \\
& (m \neq 0,1), \\
A_{m}^{\mathrm{s}}= & B_{m}^{\mathrm{s}}=-C_{m}^{\mathrm{s}}=-D_{m}^{\mathrm{s}}=-\frac{\kappa c_{m}^{\mathrm{c}} R}{2} \\
& (m \neq 0,1),
\end{aligned}
$$

where $\kappa=k /\left\{2\left(\eta^{(\circ)}+\eta^{(\mathrm{i})}\right)\right\}$. From Eqs. (6), (7) and (14), flow profiles are obtained as

$$
\begin{aligned}
& v_{r}^{(\mathrm{o})}=-\sum_{m=1}^{\infty} \frac{\kappa m\left[\left(\frac{R}{r}\right)^{m-1}-\left(\frac{R}{r}\right)^{m+1}\right]}{2} \\
& v_{r}^{(\mathrm{i})}=-\sum_{m=1}^{\infty} \frac{\kappa m\left[\left(\frac{r}{R}\right)^{m+1}-\left(\frac{r}{R}\right)^{m-1}\right]}{2} \\
& v_{\theta}^{(\mathrm{o})}=\left.-2 \frac{B_{0}^{\mathrm{i})}}{R^{2}} r \cos m \theta+c_{m}^{\mathrm{s}} \sin m \theta\right), \\
&-\sum_{m=1}^{\infty} \frac{\kappa\left[(m-2)\left(\frac{R}{r}\right)^{m-1}-m\left(\frac{R}{r}\right)^{m+1}\right]}{2} \\
& v_{\theta}^{(\mathrm{i})}=-2 \frac{B_{0}^{(\mathrm{i})}}{R^{2}} r \\
&\left.-\sum_{m=1}^{\infty} \frac{\kappa\left[-(m+2)\left(\frac{r}{R}\right)^{m+1}+m\left(\frac{r}{R}\right)^{m-1}\right]}{2} \sin m \theta\right), \\
&\left(c_{m}^{\mathrm{c}} \sin m \theta-c_{m}^{\mathrm{s}} \cos m \theta\right), \\
&\left(_{m}^{\mathrm{c}} \sin m \theta-c_{m}^{\mathrm{s}} \cos m \theta\right),
\end{aligned}
$$


and the tangential flow at the interface $(r=R), u(\theta)$, is obtained as

$$
u(\theta)=-2 \frac{B_{0}^{(\mathrm{i})}}{R^{2}} R+\kappa \sum_{m=1}^{\infty}\left(c_{m}^{\mathrm{c}} \sin m \theta-c_{m}^{\mathrm{s}} \cos m \theta\right) .
$$

As the particle is a solid, the flow velocity on the particle is $\mathbf{0}$ (no-slip condition). Assuming that the particle size is small enough, this condition can be described as $u(0)=0$. Using this condition, $B_{0}^{(\mathrm{i})}$ is determined as

$$
B_{0}^{(\mathrm{i})}=-\frac{\kappa R^{2}}{2} \sum_{m=1}^{\infty} \frac{c_{m}^{\mathrm{s}}}{R}
$$

At infinity, $v_{r}^{(\mathrm{o})}=-\kappa\left(c_{1}^{\mathrm{c}} \cos \theta+c_{1}^{\mathrm{s}} \sin \theta\right) / 2$ and $v_{\theta}^{(\mathrm{o})}=-2 \frac{B_{0}^{(\mathrm{i})}}{R^{2}} r-\kappa\left(-c_{1}^{\mathrm{c}} \sin \theta+c_{1}^{\mathrm{s}} \cos \theta\right) / 2$, which means the droplet translational velocity $(\boldsymbol{V})$ and the angular velocity in the laboratory frame $(\Omega)$ are

$$
\begin{aligned}
& \boldsymbol{V}=\kappa \frac{c_{1}^{\mathrm{c}}}{2} \boldsymbol{e}_{x}+\kappa \frac{c_{1}^{\mathrm{s}}}{2} \boldsymbol{e}_{y}, \\
& \Omega=2 \frac{B_{0}^{(\mathrm{i})}}{R^{2}}=-\kappa \sum_{m=1}^{\infty} \frac{c_{m}^{\mathrm{s}}}{R} .
\end{aligned}
$$

$\boldsymbol{e}_{x}$ and $\boldsymbol{e}_{y}$ in Eq. (18) are the unit vectors in the direction of $x$ axis $(\theta=0)$ and $y$ axis $(\theta=\pi / 2)$, respectively. The calculated flow fields are illustrated in the case of translational motion and in the case of rotational motion using Line Integral Convolution [9] in Fig. 1 (b). It is noted that the rotational speed of a droplet is proportional to the sum of the Fourier coefficients corresponding to an antisymmetric concentration field about the anterior-posterior axis.

\section{ANALYSIS OF THE MODEL}

Using Eq. (3), we calculate the velocity and the angular velocity of the droplet in this section. Since higher Fourier modes of $c$ decay quickly due to diffusion, we neglect $c_{n}^{\mathrm{c}}$, and $c_{n}^{\mathrm{s}}$ for $|n| \geq 2$. Letting $c_{0}^{\mathrm{c}}, c_{1}^{\mathrm{c}}$, and $c_{1}^{\mathrm{s}}$ be replaced by $X, Y$ and $Z$, Eq. (3) is described as

$$
\begin{aligned}
\frac{\mathrm{d} X}{\mathrm{~d} t} & =-\alpha\left(X-X_{0}\right)+\frac{\kappa}{R}\left\{Y^{2}-\left(Y_{0}\right)^{2}+Z^{2}\right\}, \\
\frac{\mathrm{d} Y}{\mathrm{~d} t} & =\lambda_{Y}\left(Y-Y_{0}\right)-\frac{\kappa}{R} Z^{2}, \\
\frac{\mathrm{d} Z}{\mathrm{~d} t} & =\lambda_{Z} Z+\frac{\kappa}{R}\left(Y-Y_{0}\right) Z .
\end{aligned}
$$


Here, $X_{0}=\kappa \beta^{2} \rho^{2} / R\left(\rho^{2}+1\right)+\beta / 2$, and $Y_{0}=\beta \rho^{2} /\left(\rho^{2}+1\right)$ are the values of $X$ and $Y$ at the fixed point at which $Z=0$. Here, $\rho=R / l_{\mathrm{d}}$ is the radius normalized by the characteristic length of diffusion, $l_{\mathrm{d}}=\sqrt{D / \alpha} \cdot \lambda_{Y}$ and $\lambda_{Z}$ are the eigenvalues for the linearized equations for $Y$ and $Z$,

$$
\begin{aligned}
& \lambda_{Y}=-\alpha\left(1+\frac{1}{\rho^{2}}\right), \\
& \lambda_{Z}=\frac{\kappa}{R} Y_{0}-\alpha\left(1+\frac{1}{\rho^{2}}\right)=\frac{\alpha \rho^{2}}{\rho^{2}+1}\left\{\frac{L}{\rho}-\left(1+\frac{1}{\rho^{2}}\right)^{2}\right\},
\end{aligned}
$$

respectively, where $L=l_{\mathrm{a}} / l_{\mathrm{d}}$ is the ratio between another characteristic length, $l_{\mathrm{a}}=$ $\kappa \beta / \alpha$, and $l_{\mathrm{d}}$. $l_{\mathrm{a}}$ is the length of advective transportation during $1 / \alpha$ since $u \sim \kappa c_{1}^{\mathrm{c}} \sim$ $\kappa \beta \rho^{2} /\left(\rho^{2}+1\right) \sim \kappa \beta$. If $\lambda_{Z}$ is positive, the fixed point, $\left(X_{0}, Y_{0}, 0\right)$, is unstable, which means a finite angular velocity of a droplet obtained from Eq. (19).

The phase diagram of the motion can be illustrated as shown in Fig. 2 (a) using two kinds of motion: translational motion and rotational motion. To realize rotation, it is necessary that the advection term in Eq. (3) is dominant; that is, $L \gtrsim 1$ is needed. In fact, $\lambda_{Z}$ can be positive only when $L>16 \sqrt{3} / 9 \approx 3.08$. When $R \lesssim l_{\mathrm{d}}(\rho \lesssim 1)$, the concentration field is almost uniform due to diffusion, so that flow is too weak to generate rotation. On the other hand, when $R \gtrsim l_{\mathrm{a}}(\rho \gtrsim L)$, the uniform decomposition term in $(3),-\alpha c$, becomes dominant and the concentration field is almost uniform. Therefore, only when $L$ is large enough and $R$ is between $R_{1}^{\mathrm{c}} \approx l_{\mathrm{d}}$ and $R_{\mathrm{u}}^{\mathrm{c}} \approx l_{\mathrm{a}}$, can the droplet spontaneously rotate due to the coupling between the mirror symmetric concentration field $\left(Y_{0}\right)$ and mirror antisymmetric concentration field $(Z)$ through the rotational flow field. Since there is no other bifurcation, there are only a pair of stable fixed points with non-zero $Z$, which are calculated as $X=3 \alpha l_{\mathrm{a}} / 2 \kappa, Y=-R \lambda_{Y} / \kappa$ and $Z= \pm R \sqrt{-\lambda_{Y} \lambda_{Z}} / \kappa$, when $R_{1}^{\mathrm{c}}<R<R_{\mathrm{u}}^{\mathrm{c}}$. Using Eq. (18) and Eq. (19), $\boldsymbol{V}$ and $\Omega$ in the steady state are calculated as

$$
\begin{aligned}
& \boldsymbol{V}= \begin{cases}\left(-\lambda_{Y} l_{\mathrm{a}}, 0\right) & \left(R<R_{\mathrm{l}}^{\mathrm{c}}, R>R_{\mathrm{u}}^{\mathrm{c}}\right) \\
\left(-\frac{\lambda_{Y} R}{2}, \pm \frac{R}{2} \sqrt{-\lambda_{Y} \lambda_{Z}}\right) & \left(R_{1}^{\mathrm{c}} \leq R \leq R_{\mathrm{u}}^{\mathrm{c}}\right)\end{cases} \\
& \Omega= \begin{cases}0 & \left(R<R_{1}^{\mathrm{c}}, R>R_{\mathrm{u}}^{\mathrm{c}}\right) \\
\mp \sqrt{-\lambda_{Y} \lambda_{Z}} & \\
=\mp \alpha \sqrt{\frac{l_{\mathrm{a}}}{R}-\left\{1+\left(\frac{l_{\mathrm{d}}}{R}\right)^{2}\right\}^{2}} & \left(R_{1}^{\mathrm{c}} \leq R \leq R_{\mathrm{u}}^{\mathrm{c}}\right)\end{cases}
\end{aligned}
$$




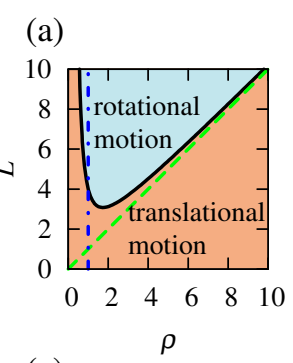

(c)

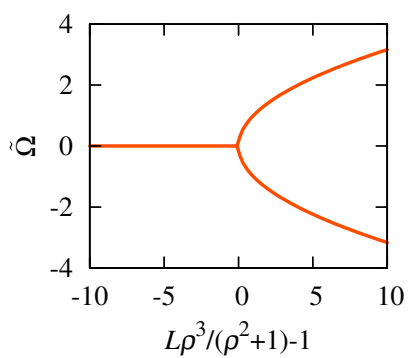

(b-1)

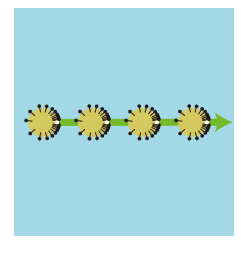

(d) (b-2)
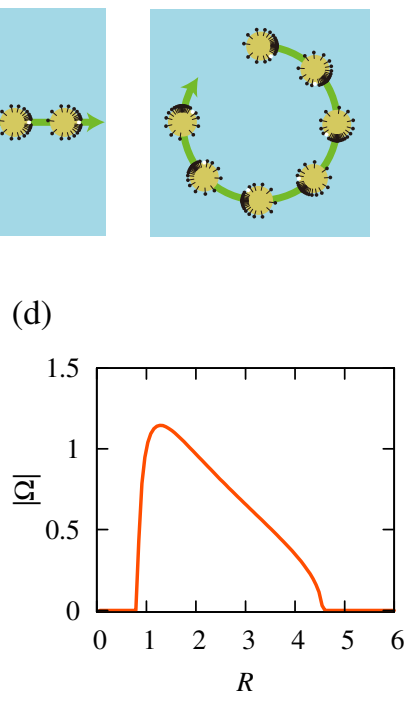

FIG. 2. (Color Online) Bifurcation of the motion of a droplet. (a) Phase diagram of the motion. One-point dashed line (blue) and dashed line (green) represent $R=l_{\mathrm{d}}$ and $R=l_{\mathrm{a}}$, respectively. (b) Schematic diagrams of the interface concentration field and the motion of a droplet in a laboratory frame. When the droplet moves straight (b-1) and rotates (b-2), the concentration field is mirror symmetric and asymmetric about the anterior-posterior axis, respectively. (c) Dependence of $\tilde{\Omega}$ on $L \rho^{3} /\left(\rho^{2}+1\right)^{2}-1$. (d) Dependence of $|\Omega|$ on $R . \alpha=1, l_{\mathrm{d}}=1$, and $l_{\mathrm{a}}=5$. With these values, $R_{1}^{\mathrm{c}}=0.83, R_{\mathrm{u}}^{\mathrm{c}}=4.55$ and $|\Omega|$ reaches a maximum value at $R=1.28$.

The interface concentration and the motion of the droplet in a laboratory frame are schematically illustrated in Fig. 2 (b). When a droplet exhibits translational motion $\left(R<R_{1}^{\mathrm{c}}, R>\right.$ $\left.R_{\mathrm{u}}^{\mathrm{c}}\right)$, the interface concentration is symmetric about the anterior-posterior axis and the anterior-posterior axis always corresponds to the direction of the motion. On the other hand, when a droplet rotates $\left(R_{1}^{\mathrm{c}}<R<R_{\mathrm{u}}^{\mathrm{c}}\right)$, the interface concentration is asymmetric about the anterior-posterior axis and the particle is always the inside of the trajectory. Considering the normalized $\Omega, \tilde{\Omega}=\Omega / \lambda_{Y}, \tilde{\Omega}$ is the square root of the distance from the critical point, $\left|L \rho^{3} /\left(\rho^{2}+1\right)^{2}-1\right|$, which corresponds to the stable solution of the normal form of pitch-fork bifurcation [10], as shown in Fig. 2 (c). Figure $2(\mathrm{~d})$ shows the dependence of $|\Omega|$ on $R$ when $l_{\mathrm{d}}, l_{\mathrm{a}}$ and $\alpha$ are fixed. $|\Omega|$ has a maximum value at a radius between $R_{1}^{\mathrm{c}}$ and $R_{\mathrm{u}}^{\mathrm{c}}$. 


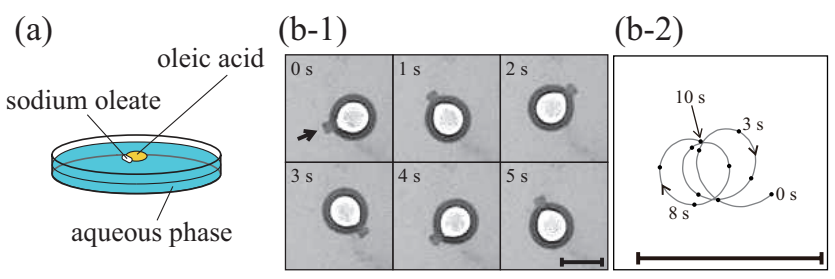

FIG. 3. (Color Online) Outline of the experimental system. (a) Schematic diagram. A petri dish with a radius of $7.5 \mathrm{~cm}$ was filled with $100 \mathrm{ml}$ water. A droplet of oleic acid together with a $3 \mathrm{~mm}$ particle of sodium oleate was placed on the water surface. The droplet moved spontaneously driven by the Marangoni effect [7]. (b) Trajectory of a droplet with a radius of $5.9 \mathrm{~mm}(300 \mu \mathrm{l})$. (b-1) Snapshots of a droplet per $1 \mathrm{~s}$. The arrow indicates the particle fixed on the interface. (b-2) Trajectory of the center of mass of the droplet. Both scale bars represent $10 \mathrm{~mm}$.

\section{COMPARISON WITH THE EXPERIMENT}

Using above theoretical results, we analyzed the corresponding experiment reported in [7] with additional data. The schematic diagram of the experimental setup is illustrated in Fig. 3 (a). One hundred milliliters of water, which was purified with a MilliQ filtering system (Millipore), was placed to a petri dish. A droplet of oleic acid (Wako Pure Chemical Industries; 159-00246) and a solid sodium oleate (soap) were floated on the aqueous phase in the petri dish. A solid column of sodium oleate (3 $\mathrm{mm}$ in length) was chosen from a commercial sample (Nacalai Tesque; 257-02). The movement of the solid/liquid composite was captured by a digital video camera at 30 frames per second at room temperature, and then analyzed using Image J (http://rsbweb.nih.gov/ij/docs/index.html).

A time series of snapshots of a droplet, and the corresponding trajectory of the center of mass of the droplet are shown in Fig. 3 (b). Due to the fluctuation of rotational speed, the trajectory does not exhibit a closed circle. From the analysis of observed trajectories, the angular velocity, $\Omega$, was measured. Time courses of $|\Omega|$ are shown in Fig. 4 (a). Five experiments were performed for each volume to yield the distribution of $|\Omega|$, which is shown in Fig. 4 (b). To determine the maximum value of the distribution, the distribution was fitted with the function, $\exp \left\{-b^{4} \Omega^{2}\left(\Omega^{2}-a\right)+c\right\}$, using the weighted nonlinear least-squares Levenberg-Marquardt algorithm, where the fitting parameters were $a, b$ and $c$ [11]. The number of events was taken as the weight for fitting. Using these parameters, the peak of the distribution, $\Omega_{\text {typ }}$, is calculated as 0 when $a<0$, and $\sqrt{a / 2}$ when $a \geq 0$. We used 
(a-1)

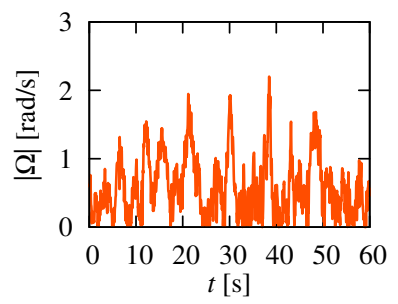

(b)

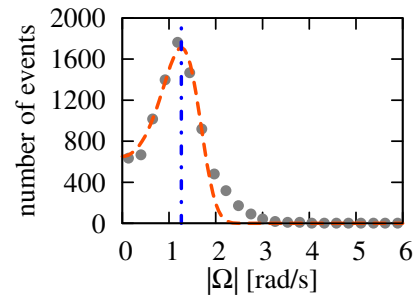

(a-2)

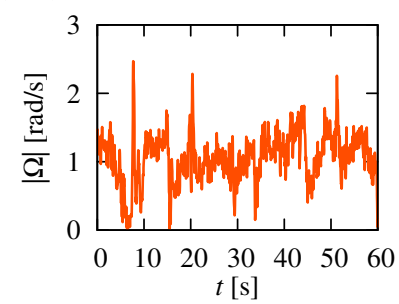

(c)

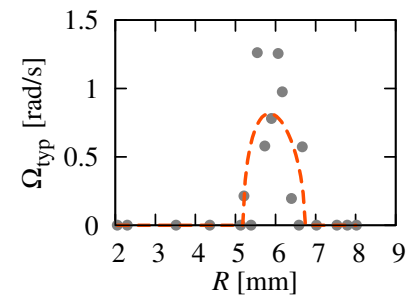

FIG. 4. (Color Online) Analysis of the experimental results. (a) Time series of the absolute value of angular velocity of a droplet with a radius of $2.3 \mathrm{~mm}(50 \mu \mathrm{l} ; \mathrm{a}-1)$ and $5.6 \mathrm{~mm}(260 \mu \mathrm{l}$; a-2). (b) Distribution of $|\Omega|$ obtained from experiments using a droplet with a radius of $5.6 \mathrm{~mm}$ (closed circles). Dashed line (orange) is the fitted curve. The parameters were estimated as $a=3.19$, $b=0.78$ and $c=6.48$. One-point dashed line (blue) represents $\Omega_{\text {typ }}=1.3 \mathrm{rad} / \mathrm{s}$. (c) Droplet size dependence of $\Omega_{\mathrm{typ}}$. Gray circles represent $\Omega_{\mathrm{typ}}$. Dashed line (orange) is the fitted curve. Estimated parameters were $\alpha=5.4 \mathrm{~s}^{-1}, l_{\mathrm{d}}=3.4 \mathrm{~mm}$ and $l_{\mathrm{a}}=10.6 \mathrm{~mm}$.

$\Omega_{\text {typ }}$ as the typical value of $\Omega$. To see the dependence of $\Omega_{\text {typ }}$ on the radius, $\Omega_{\text {typ }}$ is plotted against the droplet radius in Fig. 4 (c). Here, the radius was estimated from the image of the droplet. As shown in Fig. 4 (c), the radius with the highest $\Omega_{\text {typ }}$ and the critical radii, $R_{1}^{\mathrm{c}}$ and $R_{\mathrm{u}}^{\mathrm{c}}$, were all observed as predicted by our theoretical considerations. By fitting Eq. (26) to the obtained points, $\left(R, \Omega_{\mathrm{typ}}\right)$, we estimated $l_{\mathrm{d}}, l_{\mathrm{a}}$ and $\alpha$. The estimated parameters of the fitted curve, which is shown in Fig. 4 (c), were $\alpha=5.4 \mathrm{~s}^{-1}, l_{\mathrm{a}}=10.6 \mathrm{~mm}$, and $l_{\mathrm{d}}=3.4 \mathrm{~mm}$. It is noted that the time scale (the period of rotation) and the space scale (the size of the droplet and the particle) in the experiment are the same order as $1 / \alpha$, and $l_{\mathrm{d}}$ and $l_{\mathrm{a}}$, respectively. So far, quantitative comparison is not possible within our model since $\alpha$ and $\beta$ is difficult to measure experimentally. 


\section{SUMMARY}

In summary, we manifested the physical mechanism of the spontaneous rotation of a mirror symmetric system consisting of a droplet and a particle attached at the interface. Using the flow field calculated with Stokes equation, we analytically found that when the Marangoni flow is strong enough, there are two critical radii of the droplet for rotation and the peak of angular velocity at a certain radius between these two critical radii. For verification, we compare our model with the experiment and these theoretical predicted results were ascertained in the experiment. Although we analyze only the spontaneous rotation driven by the Marangoni effect in this article, the advective transport of objects is generally observed in self-propelled phenomena such as droplets driven by any propulsive force and amoebae cells. Therefore, the advective nonlinearity similar to that in this article is expected to cause the symmetry breaking for rotation in various kinds of self-propelled phenomena.

\section{ACKNOWLEDGEMENT}

The authors thank T. Ohta, K. Yoshikawa, M. Sano, and H. Kori for their helpful discussion. K. H. N. is supported by a JSPS fellowship for young scientists (No.23-1819). This study was supported by Grants-in-Aid for Young Scientists (B) (No.21740282) to H. K. and (No.23740317) to N. Y.

[1] S. Ramaswamy, Annu. Rev. Cond. Mat. Phys. 1, 323 (2010); P. Hänggi, Rev. Mod. Phys. 81, 387 (2009).

[2] J. Toner, Y. Tu, and S. Ramaswamy, Ann. Phys. 318, 170 (2005).

[3] K. Nagai, Y. Sumino, H. Kitahata, and K. Yoshikawa, Phys. Rev. E 71, 065301(R) (2005); K. Nagai, Y. Sumino, and K. Yoshikawa, Colloid Surf. B 56, 197 (2007); H. Kitahata, N. Yoshinaga, K. H. Nagai, and Y. Sumino, Phys. Rev. E 84, 015101(R) (2011).

[4] T. Toyota, N. Maru, M. M. Hanczyc, T. Ikegami, and T. Sugawara, J. Am. Chem. Soc. 131, 5012 (2009); S. Thutupalli, R. Seemann, and S. Herminghaus, New J. Phys. 13, 073021 (2011). 
[5] N. J. Suematsu, S. Nakata, A. Awazu and H. Nishimori, Phys. Rev. E 81, 056210 (2010); S. Soh, K. Bishop, and B. Grzybowski, J. Phys. Chem. B 112, 10848 (2008).

[6] S. Yabunaka, T. Ohta, and N. Yoshinaga, J. Chem. Phys. 136, 074904 (2012); N. Yoshinaga, K. H. Nagai, Y. Sumino, and H. Kitahata, Phys. Rev. E 86, 016108 (2012).

[7] F. Takabatake, N. Magome, M. Ichikawa, and K. Yoshikawa, J. Chem. Phys. 134, 114704 (2011).

[8] N. O. Young, J. S. Goldstein, and M. J. Block, J. Fluid Mech. 6, 350 (1959).

[9] B. Cabral and L. C. Leedom, ACM Computer Graphics (Proceedings Siggraph) , 263 (1993).

[10] J. Guckenheimer and P. Holmes, Nonlinear Oscillations, Dynamical Systems and Bifurcations of Vector Fields (Springer-Verlag, Berlin, 1990).

[11] K. Levenberg, Quart. Appl. Math. 2, 164-168 (1944). 